\title{
RADIOCARBON LABORATORIES
}

This is Radiocarbon's annual list of active radiocarbon laboratories and personnel known to us. Conventional beta-counting facilities are listed in Part I, and accelerator mass spectrometry (AMS) facilities are listed in Part II. Laboratory code designations, used to identify published dates, are given to the left of the listing. (See p 501 for a complete list of past and present lab codes.)

Please notify us of any changes in staff, addresses, or other contact information.

\section{CONVENTIONAL ${ }^{14} \mathrm{C}$ COUNTING FACILITIES}

\section{ARGENTINA}

$\mathrm{AC}$

Héctor Osvaldo Panarello

Pabellón INGEIS

Ciudad Universitaria

1428 Buenos Aires, Argentina

Tel: +54 114783 3021/23; Fax: +54 1147833024

Email: hector@ingeis.uba.ar

http://www.ingeis.uba.ar/

LP Jorge E. Carbonari and Roberto A. Huarte

Laboratorio de Tritio y Radiocarbono, LATYR

Centro de Investigaciones Geológicas, CIG

Facultad de Ciencias Naturales y Museo, UNLP

Paseo del Bosque s/n. (1900) La Plata, Argentina

Tel: + 542214270648

Email: latyr@fcnym.unlp.edu.ar

\section{AUSTRIA}

IAEA Manfred Gröning

International Atomic Energy Agency (IAEA)

Isotope Hydrology Laboratory

Wagramerstrasse 5

P.O. Box 100

A-1400 Vienna, Austria

Tel: +43 12600 21740/21766; Fax: +43 126007

Email: M.Groening@iaea.org

http://www.iaea.org/programmes/rial/pci/isotopehydrology/

Roland Tesch

ARC Seibersdorf research $\mathrm{GmbH}$

Biogenetics - Natural Resources c/o Arsenal Research

Faradaygasse 3, Arsenal Objekt 214

A-1030 Wien, Austria

Tel: +43/(0)50550 - 6516; Fax: +43/(0)50550 - 6587

Email: roland.tesch@arcs.ac.at

http://www.arcs.ac.at

\section{BELGIUM}

IRPA M. Van Strydonck

Royal Institute for Cultural Heritage

Jubelpark 1

B-1000 Brussels, Belgium

Tel: +32 27396711 (institute), +32 27396702 (lab)

Fax: +32 27320105

Email: mark.vanstrydonck@kikirpa.be 


\section{BELARUS}

IGSB N. D. Michailov

Institute of Geochemistry and Geophysics of the National

Academy of Sciences of Belarus

Kuprevich str. 7

Minsk 220141, Belarus

Tel: +375 (17) 26381 13; Fax: +375 (17) 2636398

Email: mihailov@igig.org.by

BRAZIL

CENA Luiz Carlos Ruiz Pessenda

Radiocarbon Laboratory

Centro de Energia Nuclear na Agricultura

Universidade de São Paulo

Avenida Centenario 303

Caixa Postal 96 - CEP 13400-970

Piracicaba, São Paulo, Brazil

Tel: +55 193429 4656; Fax: +55 1934294610

Email: pessenda@cena.usp.br

http://www.cena.usp.br/labs/labc14.htm

FZ M. F. Santiago

Departamento de Física - UFC

Campus do Pici - Cx. Postal 6030

60455-760 Fortaleza-CE, Brazil

Tel: +55 8540089913 ; Fax: +55 8540089450

Email: marlucia@ fisica.ufc.br

\section{CANADA}

BGS Howard Melville

Department of Earth Sciences

Brock University

500 Glenridge Avenue

St. Catharines, Ontario L2S 3A1, Canada

Tel: +1 905688 5550, ext. 3522; Fax: +1 9056829020

Email: hmelvill@brocku.ca

http://www.brocku.ca/earthsciences/radiocarbon/

UL Guillaume Labrecque

Laboratoire de datation au Carbone-14 (also prepare $\mathrm{CO}_{2}$ tubes for AMS)

Centre d'études nordiques

Université Laval

Cité Universitaire

Québec, Québec G1K 7P4, Canada

Tel: +1 418656 2131, ext. 4486; Fax: +1 4186563960

Email: guillaume.labrecque@ cen.ulaval.ca

http://www.cen.ulaval.ca/english/labradio.html

CHINA

CG Yijian Chen and G. Peng

Radiocarbon Laboratory

Institute of Geology

State Seismological Bureau

P.O. Box 634

Beijing 100029, China

HL Yunzhang Yue

Second Institute of Oceanography

State Oceanic Administration

P.O. Box 1207

Hangzhou, Zheijiang 310012, China

Tel: +86 571 8076924, ext. 328; Fax: +86 5718071539 
PKU Wu Xiaohong

Archaeometry \& Archaeological Dating Laboratory

Peking University

Beijing 100871, China

Email:wuxh@pku.edu.cn

XLLQ Zhou Weijian

Institute of Earth Environment

XiYing Lu 22-2

710054 Xi'an, Shaanxi, China

Tel: +86 295512264 (work), +86 295256429 (home); Fax: +86 295522566

Email: weijian@loess.llqg.ac.cn and weijian@public.xa.sn.cn

Qiu Shua

Radiocarbon Laboratory

Institute of Archaeology, CASS

27 Wangfujing Dajie

Beijing 100710, China

Tel: +86010 65135532; Fax: +8601065135532

Li Xingguo

Institute of Vertebrate Paleontology and Paleoanthropology

Academica Sinica

P.O. Box 643

100044 Beijing, China

http://www.ivpp.ac.cn/

Jinghang Wang

Beijing Nuclear Instrument Factory

42 Donghuan Beilu, Jianguomen Wei

100020 Beijing, China

Tel: +(010) 65062266 or +(010) 65 015198; Fax: +(010) 65953749

Dai Kaimei

Department of Physics

Nanjing University

Nanjing 210024, China

Tel: +86 25 3596746; Fax: +86 25307965

Email: postphys@nju.edu.cn

Wang Jian

Department of Geography

Nanjing Normal University

Nanjing 210093, China

Tel: +86 25 3303666, ext. 3202; Fax: +86 253307448

Email: jwang@njnu.edu.cn

Gao Zhonghe or Chen Xiaoming

Seismological Bureau of Jiangsu Province

3 Weigang

Nanjing 210014, China

Tel: +86 25 4432919, ext. 3028; Fax: +86 254432585

Ruan Chengwen, Director

Seismological Bureau of Xinjiang Uygur Autonomous Region

42 South Beijing Road

Urumqi, Xinjiang 830011, China

Tel: +86 991 3838126; Fax: +86 9913835623

Email: xjdzj@mail.wl.xj.cn

Shen Chengde

Guangzhou Institute of Geochemistry

Chinese Academy of Sciences

Wushan, Guangzhou 510640, China

Tel: +86 20 85290062; Fax: +862085290130

Email: cdshen@gig.ac.cn 
Huang Qi

Radiocarbon Laboratory, Institute of Salt Lakes

Academia Sinica

6 Xiying Road

710043 Xi'an, Shaanxi Province, China

Tel: +86295520397

\section{CROATIA}

Z Drs. Bogomil Obelić and Nada Horvatinčić

Ruđer Bošković Institute

P. O. Box 180, Bijenička 54

10001 Zagreb, Croatia

Tel: +385 14680 219; Fax: +385 14680239

Email: Bogomil.Obelic@irb.hr and Nada.Horvatincic@irb.hr

http://www.irb.hr/en/str/zef/z3labs/lna/

\section{CZECH REPUBLIC}

CRL Ivo Svetlik and Dagmar Dreslerova

CRL Radiocarbon Laboratory

Na Truhlarce 39/64

CZ-180 86 Prague 8, Czech Republic

Tel: +420 266177233; Fax: +420283842788

Email: svetlik@ujf.cas.cz and dreslerova@arup.cas.cz

CU Jiří Bruthans

Department of Hydrogeology

Charles University

Albertov 6

CZ-128 43 Prague 2, Czech Republic

Tel: +42(0) 221951566 or +42(0) 221 951111; Fax: +42(0) 221951556

Email: brutus@natur.cuni.cz

\section{DENMARK}

K Kaare Lund Rasmussen

${ }^{14} \mathrm{C}$ Dating Laboratory

National Museum

Ny Vestergade 11

DK-1471 Copenhagen K, Denmark

Tel: +45 3347 3176; Fax: +45 33473310

Email: kaare.lund.rasmussen@natmus.dk

\section{EGYPT}

IFAO Mohammed Mahran

Institut français d'archéologie orientale (IFAO)

Laboratoire de datation par le radiocarbone

37, rue al-Cheikh Ali Youssef

P.O. Box 11562 Qasr al-ainy

11421 Cairo, Egypt

Tel: +202 7971 615/600; Fax: +20279 44635

Email: c14@ifao.egnet.net

http://www.ifao.egnet.net/c14

\section{ESTONIA}

Ta Volli Kalm and Arvi Liiva

Radiocarbon Laboratory

Institute of Geology

University of Tartu

Vanemuise St. 46

51014 Tartu, Estonia

Tel/Fax: +372 7375836

Email: volli.kalm@ut.ee and geol@ut.ee 
Enn Kaup

Radiocarbon Laboratory

Institute of Geology at Tallinn University of Technology

Estonia pst 7

10143 Tallinn, Estonia

Tel: +372 645 4679; Fax: +372631 2074

Email:kaup@gi.ee

Jaan-Mati Punning

Institute of Ecology

Kevade 2

10137 Tallinn, Estonia

Tel: +372 2451 634; Fax: +3722453748

Email:mati@eco.edu.ee

\section{FINLAND}

Hel Markku Oinonen

Dating Laboratory

P.O. Box 64

FIN-00014 Helsinki University, Finland

Tel: +3589191 50740; Fax: +358919150741

Email: markku.j.oinonen@helsinki.fi

\section{FRANCE}

Gif Michel Fontugne

Centre des Faibles Radioactivités

Laboratoire mixte CNRS-CEA

F-91198 Gif-sur-Yvette Cedex, France

Tel: +33 1698235 25; Fax: +33 169823568

Email: Michel.Fontugne@1sce.cnrs-gif.fr

and

Laboratoire Souterrain de Modane

Laboratoire mixte IN2I 3-CNRS/DSM-CEA

90, Rue Polset

F-73500 Modane, France

http://www.cnrs.fr

Ly Christine Oberlin

CDRC - Centre de Datation par le RadioCarbone UMR 5138 CNRS

Université Claude Bernard Lyon I

40, Boulevard Niels Bohr

F-69622 Villeurbanne Cedex, France

Tel: +33472 4482 57; Fax: +33472431317

Email: christine.oberlin@univ-lyon1.fr

http://carbon14.univ-lyon1.fr

\section{GEORGIA}

$\mathrm{TB}$

S. Pagava

Radiocarbon and Low-Level Counting Section

Nuclear Research Laboratory

I. Javakhishvili Tbilisi State University

I. Chavchavadze Av., 3

Tbilisi 0128, Georgia

Tel: +995 32 222105, +995 32 252776; Fax: +995 32252776

Email: spagava@access.sanet.ge

\section{GERMANY}

Bln

Jochen Görsdorf

Deutsches Archäologisches Institut

Zentrale, Referat Naturwissenschaften

Postfach 330014, D-14191 Berlin, Germany

Tel: +49 018887711 339; Fax: +49 01888107711339

Email: 14c@dainst.de 
HAM Peter Becker-Heidmann

Institut für Bodenkunde

Universität Hamburg

Allende-Platz 2

D-20146 Hamburg, Germany

Tel: +49 4042838 2003; Fax: +49 40428382024

Email: P.Becker-Heidmann@ifb.uni-hamburg.de

http://www.geowiss.uni-hamburg.de/i-boden/tt14c.htm

Hd Bernd Kromer

Heidelberger Akademie der Wissenschaften

c/o Institut für Umweltphysik

Universität Heidelberg

Im Neuenheimer Feld 229

D-69120 Heidelberg, Germany

Tel: +49 6221546 357; Fax: +49 6221546405

Email: Bernd.Kromer@iup.uni-heidelberg.de

Hv Mebus A. Geyh

Niedersächsisches Landesamt für Bodenforschung

Postfach 510153

D-30655 Hannover-Stillweg 2, Germany

Tel: +49 511643 2537; Fax: +49 5116432304

Email: Mebus.Geyh@t-online.de

KI Helmut Erlenkeuser and Pieter M. Grootes

Leibniz-Labor

Christian-Albrechts-Universität

Max-Eyth-Str. 11-13

D-24118 Kiel, Germany

Tel: +49 4318803894 (P.M.G.), +49 4318803896 (H.E.)

Fax: +49 4318807401

Email: pgrootes@ @eibniz.uni-kiel.de and herlenkeuser@ leibniz.uni-kiel.de http://www.uni-kiel.de/leibniz/

$\mathrm{KN}$

Bernhard Weninger

Labor für ${ }^{14} \mathrm{C}$-Datierung

Institut für Ur-und Frühgeschichte

Universität zu Köln

Weyertal 125

D-50923 Köln, Germany

Tel: +49 2214702880 / 2881; Fax: +49 2214704892

Email: b.weninger@uni-koeln.de

http://www.calpal.de

LZ Achim Hiller

UFZ-Umweltforschungszentrum Leipzig-Halle $\mathrm{GmbH}$

Sektion Hydrogeologie

Arbeitsgruppe Paläoklimatologie

Theodor-Lieser-Strasse 4

D-06120 Halle, Germany

Tel: +49 3455585 226; Fax: +49 3455585559

Email: a.hiller@hlug.de

\section{GREECE}

DEM Yannis Maniatis

Laboratory of Archaeometry

Institute of Materials Science

National Centre for Scientific Research "Demokritos"

15310 Aghia Paraskevi

Attiki, Greece

Tel: +30 16503389 or +301 6524821; Fax: +30 16519430

Email: maniatis@ims.demokritos.gr

http://www.ims.demokritos.gr/archae 
LIH Nicolaos Zouridakis

Laboratory of Isotope Hydrology

Institute of Physical Chemistry

National Centre for Scientific Research "Demokritos"

15310 Aghia Paraskevi Attikis

POB 60228, Greece

Tel: +30 1 6503969; Fax: +30 16511766

Email: nizouri@chem.demokritos.gr

\section{HUNGARY}

Deb Zsusa Szántó

Institute of Nuclear Research of the Hungarian Academy of Sciences

H-4026 Bem tér 18/c

P.O. Box 51

H-4001 Debrecen, Hungary

Tel: +36 52 417266; Fax: +36 52416181

Email: aszanto@namafia.atomki.hu

\section{ICELAND}

Páll Theodórsson

Science Institute

University of Iceland

Dunhaga 3

IS-107 Reykjavík, Iceland

Tel: +354 525 4800; Fax: +354 5528911

Email: pth@raunvis.hi.is

INDIA

BS Dr. Supriyo Chakraborty

Radiocarbon Dating Laboratory

Birbal Sahni Institute of Palaeobotany

53 University Road

Lucknow 226007 , India

Tel: +91 522274 0008; Fax: +91 5222740485

Email: c14_bsip@yahoo.com and supriyoc@gmail.com

JUBR S. D. Chatterjee, R. C. Sastri, and Haradhan De

Biren Roy Research Laboratory for Archaeological Dating

Department of Physics

Jadavpur University

Calcutta 700 032, India

Tel: +91 33473 4044; Fax: +91 334734266

PRL Dr. M. G. Yadava

Radiocarbon Dating Laboratory

Planetary and Geosciences Division

Physical Research Laboratory

Navrangpura

Ahmedabad 380 009, India

Tel: +91 79 26314367; Fax: +91 7926301502

Email: myadava@prl.res.in

PRLCH R. Bhushan

Physical Research Laboratory

Chemistry Department

Planetary and Geosciences Division

Navrangpura

Ahmedabad 380 009, India

Tel: +91 79 2631415; Fax: +91 7926301502

Email: bhushan@prl.ernet.in 


\section{INDONESIA}

Wisjachudin Faisal

Staff of Research and Development Center for Advance Technology

National Nuclear Energy Agency

Jl. Babarsari, P.O. Box 1008

Yogyakarta 55101, Indonesia

Tel: +62 274 515435; Fax: +62 274561824

Email: p3tm@ indo.net.id and wisya @batan.go.id

\section{IRELAND}

UCD Peter I. Mitchell and Edward McGee

UCD Radiocarbon Laboratory

Department of Experimental Physics

University College Dublin

Belfield, Dublin 4, Ireland

Tel: +353 1716 2220; Fax: +353 12837275

Email: Peter.Mitchell@ucd.ie and Edward.Mcgee@ucd.ie

http://www.ucd.ie/radphys/page2.htm

ISRAEL

RT Elisabetta Boaretto

Radiocarbon Dating and Cosmogenic Isotopes Laboratory

Weizmann Institute of Science

76100 Rehovot, Israel

Tel: +972 893 43213; Fax: +972 89346062

Email: Elisabetta.Boaretto@wis.weizmann.ac.il

\section{ITALY}

ENEA Paolo Bartolomei and Giuseppe Magnani

ENEA C.R. "E. Clementel"

Via don Fiammelli, 2

I-41028 Bologna, Italy

Tel: +39 51 6098168; Fax: +3951 6098187

Email: paolo.bartolomei@bologna.enea.it and giuseppe.magnani@bologna.enea.it

http://diaf3.bologna.enea.it/

$\mathrm{R}$

Salvatore Improta

Dipartimento di Fisica

Università "La Sapienza"

Piazzale Aldo Moro, 2

I-00185 Rome, Italy

Tel: +39 6 49914208; Fax: +3964957697

Email: Salvatore.Improta@ roma1.infn.it

and

Prof. Giorgio Belluomini

Istituto per le Tecnologie Applicate ai Beni Culturali

C.N.R. Area della Ricerca di Roma

Via Salaria Km 29,3 - C.P. 10

I-00016 Monterotondo St., Rome, Italy

Tel: +3906 90672469/279

Email: giorgio.belluomini@mlib.cnr.it

Rome Gilberto Calderoni

Department of Earth Sciences

University of Rome "La Sapienza"

Piazzale Aldo Moro, 5

I-00185 Rome, Italy

Tel: +39 6499 14580; Fax: +39 649914578

Email: gilberto.calderoni@uniroma1.it 
JAPAN

IAA Takao Matsui

Shirakawa Radiometric Laboratory

Institute of Accelerator Analysis Ltd.

6-270 Ichiridan, Shirasaka, Shirakawa City

Fukushima 961-0835, Japan

Tel: +81 248211055

Email: office@iaa-ams.co.jp

http://www.iaa-ams.co.jp

KEEA Yoshimasa Takashima

Kyushu Environmental Evaluation Association

1-10-1, Matsukadai, Higashiku

Fukuoka 813-0004, Japan

Tel: +81 92662 0410; Fax: +81 926620990

Email: kawamura@keea.or.jp

NU Kunio Omoto

Radiocarbon Dating Laboratory

Department of Geography

College of Humanities and Science

Nihon University

25-40, 3 Chome, Sakurajosui

Setagaya-ku, Tokyo 156, Japan

Tel: +81 35317 9273; Fax: +81 353179429

Email: omoto@chs.nihon-u.ac.jp

OR Setsuko Shibata

Research Center of Radioisotopes

Frontier Science Innovation Center

University of Osaka Prefecture

1-2, Gakuen-cho, Sakai 599-8570, Japan

Tel: +81 72236 2221; Fax: +81 722549938

Email: shibata@ riast.osakafu-u.ac.jp

PAL Shigemoto Tokunaga

Radiocarbon Laboratory

Palynosurvey Co.

Nissan Edobashi Bld

1-10-5 Honcho, Nihonbashi

Chuoku, Tokyo, Japan

Tel: (office): +81 33241 4566; (lab) +81 274428129

Fax: +81 332414597

Email: office@palyno.co.jp

http://www.palyno.co.jp/

PLD Hideki Yamagata

Paleo Labo Co., Ltd.

63, Shima 5-chome Oguma-cho

Hashima, Gifu 501-6264, Japan

Email:pal@usiwakamaru.or.jp

http://www.paleolabo.jp/

TK

Dr. Kunio Yoshida

C-14 Dating Laboratory

The University Museum

The University of Tokyo

3-1 Hongo 7-chome

Bunkyo-ku, Tokyo 113-0033, Japan

Tel: +81 35841 2822; Fax: +81 358418450

Email: gara@um.u-tokyo.ac.jp 


\section{KOREA}

KCP Hyung Tae Kang and Kyung Yim Nah

Archaeological Studies Division

National Cultural Property Research Institute

1-57 Sejongno Chongno-gu

Seoul, Korea 110050

Tel: +82 2735 5281, ext. 323; Fax: +82 27356889

Email: vvyckht1@chollian.net

\section{LATVIA}

Riga V. S. Veksler and A. A. Kristin

Institute of Science - Application Research

Riga 50 Merkelya 11

Riga 226 050, Latvia

Tel: +371 7212501 or +3717213636

\section{LITHUANIA}

Group of Dendroclimatology and Radiometrics

Environmental Research Centre

Faculty of Natural Sciences

Vytautas Magnus University

Z.E. Zlilibero 2, LT-3018

Kaunas, Lithuania

Tel: +370 37390955

Email: atcentre@lycos.com and atc@gmf.vdu.lt

Rimantas Petrosius

Radioisotope Laboratory

Institute of Geology

Sevcenkos 13

Vilnius 2600, Lithuania

Tel: +370 2 236103; Fax: +370 2236710

Email: petros@geologin.lt

\section{MEXICO}

IMTA Israel Mata

Instituto Mexicano de Tecnología del Agua (IMTA)

Laboratorio de carbono 14

Paseo Cuauhnáhuac 8532

Col. Progreso, Jiutepec, Morelos 62550, México

Tel: +52 7773293600 Ext: 176; Fax: +52 777329368

UNAM Laura Beramendi-Orosco and Galia González-Hernández

Laboratorio Universitario de Radiocarbono

Instituto de Geofísica, Instituto de Investigaciones Antropológicas

Universidad Nacional Autónoma de México

Ciudad Universitaria, México City 04510, México

Tel: +525556229680

Email: laura@geofisica.unam.mx; galia@geofisica.unam.mx

http://geologia.igeolcu.unam.mx/LUR/Index.html

\section{MONACO}

IAEA- Dr. Laval Liong Wee Kwong

MEL International Atomic Energy Agency

Marine Environmental Laboratory

4 Quai Antoine $1^{\mathrm{er}}$

MC-98012 Monaco

Tel: +377979 77272; Fax: +377979 77273

Email: L.Liong@iaea.org 


\section{THE NETHERLANDS}

GrN J. van der Plicht

Centre for Isotope Research

University of Groningen

Nijenborgh 4

NL-9747 AG Groningen, the Netherlands

Tel: +31 50 3634760; Fax: +31 503634738

Email: j.van.der.plicht@rug.nl

\section{NEW ZEALAND}

Wk A. G. Hogg and F. Petchey

Radiocarbon Dating Laboratory

University of Waikato

Gate 9, Hillcrest Rd.

Hamilton, New Zealand

Tel: +64 7838 4278; Fax: +64 78384192

Email: alan.hogg@waikato.ac.nz and f.petchey@waikato.ac.nz

http://www.radiocarbondating.com

\section{NORWAY}

T Steinar Gulliksen

National Laboratory for ${ }^{14} \mathrm{C}$ Dating

Norwegian University of Science and Technology

N-7491 Trondheim, Norway

Tel: +47 73 593310; Fax: +47 73593383

Email: Steinar.Gulliksen@vm.ntnu.no

POLAND

Gd Anna Pazdur

Radiocarbon Laboratory

Silesian University of Technology

Institute of Physics

Krzywoustego 2

PL-44-100 Gliwice, Poland

Tel: +48 32 2372254; Fax: +48 322372488

Email: Anna.Pazdur@polsl.pl

KR

Tadeusz Kuc

Kraków Radiocarbon Laboratory

AGH University of Science and Technology

Environmental Physics Department

PL-30-059 Kraków, Poland

Tel: +48 126172979 or 6333740; Fax: +48 126340010

Email: kuc@novell.ftj.agh.edu.pl

LOD Paweł Trzeciak and Ireneusz Borowiec

Radiochemical Laboratory

Archaeological and Ethnographical Museum in Łódź

Pl. Wolnści 14

PL-91-415 Łódź, Poland

Tel: +48 426328440 or +48426334307

Fax: +48426329714

Email: jotmol@ krysia.uni.lodz.pl

\section{PORTUGAL}

$\mathrm{Sac}$

A. M. Monge Soares

Laboratório de Isótopos Ambientais

Instituto Tecnológico e Nuclear

Estrada Nacional 10

2686-953 Sacavém, Portugal

Tel: +351 2199461 80; Fax: +351219941455

Email: amsoares@itn.pt 


\section{REPUBLIC OF CHINA}

NTU

Tsung-Kwei Liu

Department of Geosciences

National Taiwan University

245 Choushan Road

Taipei, Taiwan, Republic of China

Tel/Fax: +886 23657380

Email: liutk@ccms.ntu.edu.tw

RUSSIA

GIN Leopold D. Sulerzhitsky

Geological Institute

Russian Academy of Sciences

Pyzhevsky Pereulok 7

Moscow 119017, Russia

Tel: +7 4952308136

Email: suler@ginras.ru

IEMAE Arkady B. Savinetsky

Severtsov Institute of Ecology and Evolution

Russian Academy of Sciences

Leninsky Prospekt 33

Moscow 119071, Russia

Fax: +7 4959545534

Email: histecol@ orc.ru

IGAN Olga A. Chichagova

Institute of Geography

Russian Academy of Sciences

Staromonetnyi Pereulok 29

Moscow 119017, Russia

Tel: +7 495230 8366; Fax: +7 4959590033

Email: ochichag@mtu-net.ru

LE Ganna I. Zaitseva

Institute of the History of Material Culture

Russian Academy of Sciences

Dvortsovaya Naberezhnaya 18

St. Petersburg 191186, Russia

Tel: +7 812311 8156; Fax: +7 8123116271

Email: ganna@mail.wplus.net

LU Khikmat A. Arslanov

Geographical Research Institute

St. Petersburg State University

Sredniy Prospect 41

St. Petersburg 193004, Russia

Tel/Fax: +7 8122187904

Email: kozyrev@mail.nevalink.ru

MAG Anatoly V. Lozhkin

Geology and Paleogeography of Cenozoic Laboratory

Northeast Interdisciplinary Scientific Research Institute

Russian Academy of Sciences, Far East Branch

16 Portovaya St.

Magadan 685000, Russia

Email: lozhkin@ neisri.magadan.ru

SOAN Lyobov Orlova

Institute of Geology and Mineralogy

Russian Academy of Sciences, Siberian Branch

Koptyug Prospekt 3

Novosibirsk 630090, Russia

Tel: +7 3833307839

Email: orlova@uiggm.nsc.ru 


\section{SENEGAL}

DK Maurice Ndeye

Institut Fondamental d'Afrique Noire (IFAN)

Université Cheikh Anta Diop

Dakar, Sénégal

Tel: +2218241652

Email: mndeye@ucad.sn

http://www.africainformation.net/ifan1.htm

\section{SLOVAKIA}

$\mathrm{Ba} \quad$ Pavel Povinec

Comenius University

Faculty of Mathematics and Physics

Mlynská dolina F1

SK-842 48 Bratislava 4, Slovakia

Email: povinec@gmail.com

\section{SOUTH AFRICA}

Pta Stephan Woodborne

Quaternary Research Dating Unit (QUADRU)

c/o Enviromentek, CSIR

P.O. Box 395

0001 Pretoria, South Africa

Tel: +27 12841 3380; Fax: +27 123491170

Email: swoodbor@CSIR.co.za

SPAIN

CSIC Antonio Rubinos

Geochronology Laboratory

Instituto de Química-Física Rocasolano - CSIC

Serrano, 119

28006 Madrid, Spain

Tel: +34 91561 9400; Fax: +34 915642431

Email: rubinos@iqfr.csic.es

UBAR Dr. Joan S. Mestres

Laboratori de Datació per Radiocarboni

Facultat de Química, 3a. Planta

Universitat de Barcelona

C/. Martí i Franquès, 1-11/Avda. Diagonal, 647

08028 Barcelona, Spain

Tel: +34 93403 4688; Fax: +34 934021233

Email: js.mestres@ub.edu

UGRA Elena Villafranca Sánchez

Laboratorio de Datación por C-14

Centro de Instrumentación Científica

Campus Fuentenueva, Ed. Mecenas

Universidad de Granada

18071 Granada, Spain

Tel: +34 58 244229; Fax: +34 58243391

Email: evs@ugr.es

\section{SWEDEN}

LuS Göran Skog

Radiocarbon Dating Laboratory

Lund University

Sölvegatan 12

SE-223 62 Lund, Sweden

Tel: +46 46222 7885; Fax: +46 462224830

Email: Goran.Skog@c14lab.lu.se 
U Ingrid U. Olsson

Department of Physics

Uppsala University

Box 530

SE-751 21 Uppsala, Sweden

Tel: +46 18471 3551; Fax: +46 184713524

Email: ingrid.olsson@fysik.uu.se

\section{SWITZERLAND}

B Thomas Stocker

Climate and Environmental Physics

Physics Institute, University of Bern

Sidlerstrasse 5

CH-3012 Bern, Switzerland

Tel: +41 3163144 64; Fax: +41 316318742

Email: stocker@climate.unibe.ch

http://www.climate.unibe.ch

\section{TURKEY}

METU Mustafa Özbakan

Radiocarbon Dating Laboratory

Middle East Technical University, Department of Physics

06531 Ankara, Turkey

Tel: +90 31221032 76; Fax: +90 3122101281

Email: ozbakan@metu.edu.tr

\section{UKRAINE}

IHME Michael Buzinny

The Marzeev Institute of Hygiene and Medical Ecology

Academy of Medical Sciences of Ukraine

50 Popudrenko St.

Kiev, 02094 Ukraine

Tel/Fax: +38 0445137074

Email: mbuz@ukr.net and buzinny@yahoo.com

http://users.ldc.net/ mbuz/c14.htm

Ki Nikolai N. Kovalyukh and Vadim V. Skripkin

National Academy of Sciences and Ministry of Extraordinary Situation of Ukraine

Institute of Environmental Geochemistry

Kiev Radiocarbon Laboratory

34a, Palladin Av.

Kiev, 03680 Ukraine

Tel: +38 044424 0060; Fax: +38 0444241465

Email: kyiv14c@ radgeo.freenet.kiev.ua

\section{UNITED KINGDOM}

GU

G. T. Cook

SUERC Radiocarbon Dating Laboratory

Scottish Universities Environment Research Centre

Scottish Enterprise Technology Park

Rankine Avenue

East Kilbride, Glasgow G75 0QF, Scotland

Tel: +44 13552 23332 or +44 13552 70136; Fax: +44 1355229898

Email: g.cook@surrc.gla.ac.uk

Q Roy Switsur

Cambridge Radiocarbon Dating Research Laboratory

Environmental Sciences Research Centre

East Road

Cambridge CB1 1PT, England

Tel: +44 1223 363271, ext. 2594

Email: vrs1@cam.ac.uk 
RCD R. L. Otlet and A. J. Walker

RCD Radiocarbon Dating

The Old Stables

East Lockinge, Wantage

Oxon OX12 8QY, England

Tel/Fax: +44 1235833667

SRR Dr. Charlotte L. Bryant

NERC Radiocarbon Laboratory

Scottish Enterprise Technology Park

Rankine Avenue

East Kilbride, Glasgow G75 0QF, Scotland

Tel: +44 1355 260037; Fax: +44 1355229829

Email: radiocarbon@ nercrcl.gla.ac.uk

http://www.gla.ac.uk/nercrcl

SWA Quentin Dresser

Department of Geography

University of Wales, Swansea

Singleton Park, Swansea

West Glamorgan SA2 8PP, Wales

Tel: +44 1792 295148; Fax: +44 1792295955

Email: P.Q.Dresser@swansea.ac.uk

E. M. Scott

Department of Statistics

University Gardens

University of Glasgow

Glasgow G12 8QW, Scotland

Tel: +44 141330 5125; Fax: +44 1413304814

Email:marian@stats.gla.ac.uk

\section{UNITED STATES}

A

Chris Eastoe

Laboratory of Isotope Geochemistry

Geosciences Department

University of Arizona

Tucson, Arizona 85721, USA

Tel: +1 520621 1638; Fax: +1 5206212672

Email: eastoe@geo.arizona.edu

Beta Darden G. Hood

Beta Analytic, Inc.

4985 SW 74 Court

Miami, Florida 33155, USA

Tel: +1 305667 5167; Fax: +1 3056630964

Email: dhood@ radiocarbon.com

GX Dana Krueger

Geochron Laboratories, A Division of Krueger Enterprises, Inc.

711 Concord Avenue

Cambridge, Massachusetts 02138, USA

Tel: +1 6178763691 ; Fax: +1 6176610148

Email: dkrueger@geochronlabs.com

ISGS Drs. Hong Wang and Keith Hackley

Radiocarbon Dating Laboratory

Illinois State Geological Survey

615 E. Peabody Drive

Champaign, Illinois 61820, USA

Tel: +1 217244 7692; Fax: +1 2172447004

Email: wang@ @isgs.uiuc.edu; hackley@ isgs.uiuc.edu

NIST George A. Klouda

NIST Low-Level Counting Laboratory

National Institute of Standards and Technology

100 Bureau Dr., Mailstop 8372

Gaithersburg, Maryland 20899-8371, USA

Tel: +1 301975 3931; Fax: +1 3014171321

Email: George.Klouda@nist.gov 


\section{$494 \quad$ Laboratories}

QL $\quad$ Emeritus Minze Stuiver

Quaternary Isotope Laboratory

Quaternary Research Center, Box 351360

University of Washington

Seattle, Washington 98195-1360, USA

Email: minze@u.washington.edu

UCI Ellen Druffel and Sheila Griffin

Radiocarbon Laboratory

Department of Earth System Science

University of California, Irvine

Croul Hall 2212

Irvine, California 92697-3100, USA

Tel: +1 9498242116 (Druffel/office)

Fax: +1 9498243478

Email edruffel@uci.edu and sgriffin@uci.edu

UGa Dr. John E. Noakes

Center for Applied Isotope Studies

The University of Georgia

120 Riverbend Road

Athens, Georgia 30602-4702, USA

Tel: +1 706542 1395; Fax: +1 7065426106

Email: jenoakes@uga.edu

http://www.cais.uga.edu

UTCAG Daniel Weinand

University of Tennessee

Center for Archaeometry and Geochronology

250 South Stadium Hall

Knoxville, Tennessee 37996-0720, USA

Tel: +1 865974 8771; Fax: +1 8659742686

Email: utcag@utk.edu

http://web.utk.edu/ anthrop/utcag/

\section{URUGUAY}

URU Cristina Ures and Roberto Bracco

Laboratorio de ${ }^{14} \mathrm{C}$

Facultad de Química

Universidad de la República

Gral. Flores 2124

Montevideo, Uruguay

Tel: +598 2924 8571; Fax: +598 29241906

Email: radquim@bilbo.edu.uy

\section{II. ${ }^{14} \mathrm{C}$ ACCELERATOR FACILITIES (AMS)}

\section{AUSTRALIA}

\section{ANSTO David Fink}

$\mathrm{OZ} \quad$ ANTARES AMS Facility

Physics Division

Australian Nuclear Science and Technology Organisation (ANSTO)

New Illawarra Road

Lucas Heights, NSW 2234, Australia

Tel: +61 29717 3048; Fax: +61 297173257

Email: fink@ansto.gov.au

http://www.ansto.gov.au/nugeo/ams/index.html

ANUA L. Keith Fifield

Department of Nuclear Physics, RSPhysSE

Australian National University

Canberra, ACT 0200, Australia

Tel: +61 26249 2095; Fax: +61 262490748

Email: keith.fifield@anu.edu.au 


\section{AUSTRIA}

VERA Eva Maria Wild

VERA-Laboratorium

Institut für Isotopenforschung und Kernphysik

Universität Wien

Währingerstrasse 17

A-1090 Vienna, Austria

Tel: +43 14277 51700; Fax: +43 142779517

Email: eva.maria.wild@univie.ac.at

http://isotopenforschung.univie.ac.at/

\section{BELGIUM}

KIK M. Van Strydonck

Royal Institute for Cultural Heritage

Jubelpark 1

B-1000 Brussels, Belgium

Tel: +32 27396711 (institute); +32 27396702 (lab)

Fax: +3227320105

Email: mark.vanstrydonck@kikirpa.be

http://www.kikirpa.be/www2/Site_irpa/En/Lab/Mat.htm

\section{CANADA}

TO

Roelf P. Beukens

IsoTrace Laboratory

University of Toronto

60 St. George Street

Toronto, Ontario M5S 1A7, Canada

Tel: +1 416978 4628; Fax: +1 4169784711

Email: roelf.beukens@utoronto.ca

http://www.physics.utoronto.ca/ isotrace/info.html

CHINA

PKUAMS Kexin Liu and Baoxi Han

Institute of Heavy Ion Physics

School of Physics, Peking University

Beijing 100871, China

Tel: +86 10 62758528; Fax: +86 1062751875

Email: kxliu@pku.edu.cn

\section{DENMARK}

AAR Jan Heinemeier

AMS ${ }^{14} \mathrm{C}$ Dating Laboratory

Dept. of Physics and Astronomy

University of Aarhus

DK-8000 Aarhus C, Denmark

Tel: +45 8942 3718; Fax: +45 86120740

Email: jh@phys.au.dk

http://www.c14.dk

\section{FRANCE}

\section{Gif A GDR Tandetron}

Domaine du CNRS

Avenue de la Terrasse, Bat. 30

F-91198 Gif-sur-Yvette Cedex, France

Tel: +33 1698239 15; Fax: +33 169823670

SacA Evelyne Cottereau

LMC14

bat 450 porte $4 \mathrm{E}$ CEA Saclay

F-91191 Gif-sur-Yvette Cedex, France

Tel: +3301690814 54; Fax: +330169081557

Email: cottereau@smac14.cea.fr 
Jean-Claude Duplessy, Director

Centre des Faibles Radioactivités

Laboratoire mixte CNRS-CEA

F-91198 Gif-sur-Yvette Cedex, France

Tel: +33 1698235 26; Fax: +33 169823568

Email: Jean-Claude.Duplessy@cfr.cnrs-gif.fr

$A M{ }^{14} C$

Maurice Arnold

Centre des Faibles Radioactivités (CEA-CNRS), Tandetron

Avenue de la Terrasse BP 1

F-91198 Gif-sur-Yvette Cedex, France

Tel: +33 1 698235 63; Fax: +33 169823670

Email: Maurice.Arnold@cfr.cnrs-gif.fr

AMS ${ }^{10} \mathrm{Be},{ }^{126} \mathrm{Al},{ }^{129} \mathrm{I}$

Françoise Yiou and Grant Raisbeck

CSNSM Bat. 108

F-91405 Campus Orsay Cedex, France

Tel: +33 1691552 64; Fax: +33 169155268

Email: yiou@csnsm.in2p3.fr and raisbeck@csnsm.in2p3.fr

\section{GERMANY}

Erl Dr. Wolfgang Kretschmer

Physikalisches Institut

Universität Erlangen-Nürnberg

Erwin-Rommel-Str. 1

D-91054 Erlangen, Germany

Tel: +49 9131 8527075; Fax: +49 913115249

Email: kretschmer@physik.uni-erlangen.de

http://www.ams-erlangen.com

J Dr. Axel Steinhof

${ }^{14} \mathrm{C}$ AMS Laboratory

Max-Planck-Institut für Biogeochemie

P.O. Box 100164

D-07701 Jena, Germany

Tel: +49 364157 6450; Fax: +49 3641577450

Email: steinhof@bgc-jena.mpg.de

http://www.bgc-jena.mpg.de

KIA Dr. Pieter M. Grootes and Dr. Marie-Josée Nadeau

Leibniz-Labor

Christian Albrechts Universität

Max-Eyth-Str. 11

D-24118 Kiel, Germany

Tel: +49 4318803894 (P.M.G.), +49 4318807390 (M.-J.N.)

Fax: +49 4318807401

Email: pgrootes@leibniz.uni-kiel.de (P.M.G.)

http://www.uni-kiel.de/leibniz/

ITALY

DSA Dr. Filippo Terrasi and Dr. Carmine Lubritto

Center for Isotopic Research on Cultural and Environmental Heritage (CIRCE)

Seconda Università di Napoli

Via Vivaldi 43

Caserta 81100, Italy

Email: filippo.terrasi@unina2.it and carmine.lubritto@unina2.it

LTL Dr. Lucio Calcagnile

CEDAD - AMS Radiocarbon Dating and IBA Facility

University of Lecce, Department of Engineering and Innovation

Via Per Monteroni

Lecce 73100 , Italy

Tel: +39 0831 507372; Fax: +390831507408

Email: Lucio.calcagnile@unile.it

http://www.cedad.unile.it 
JAPAN

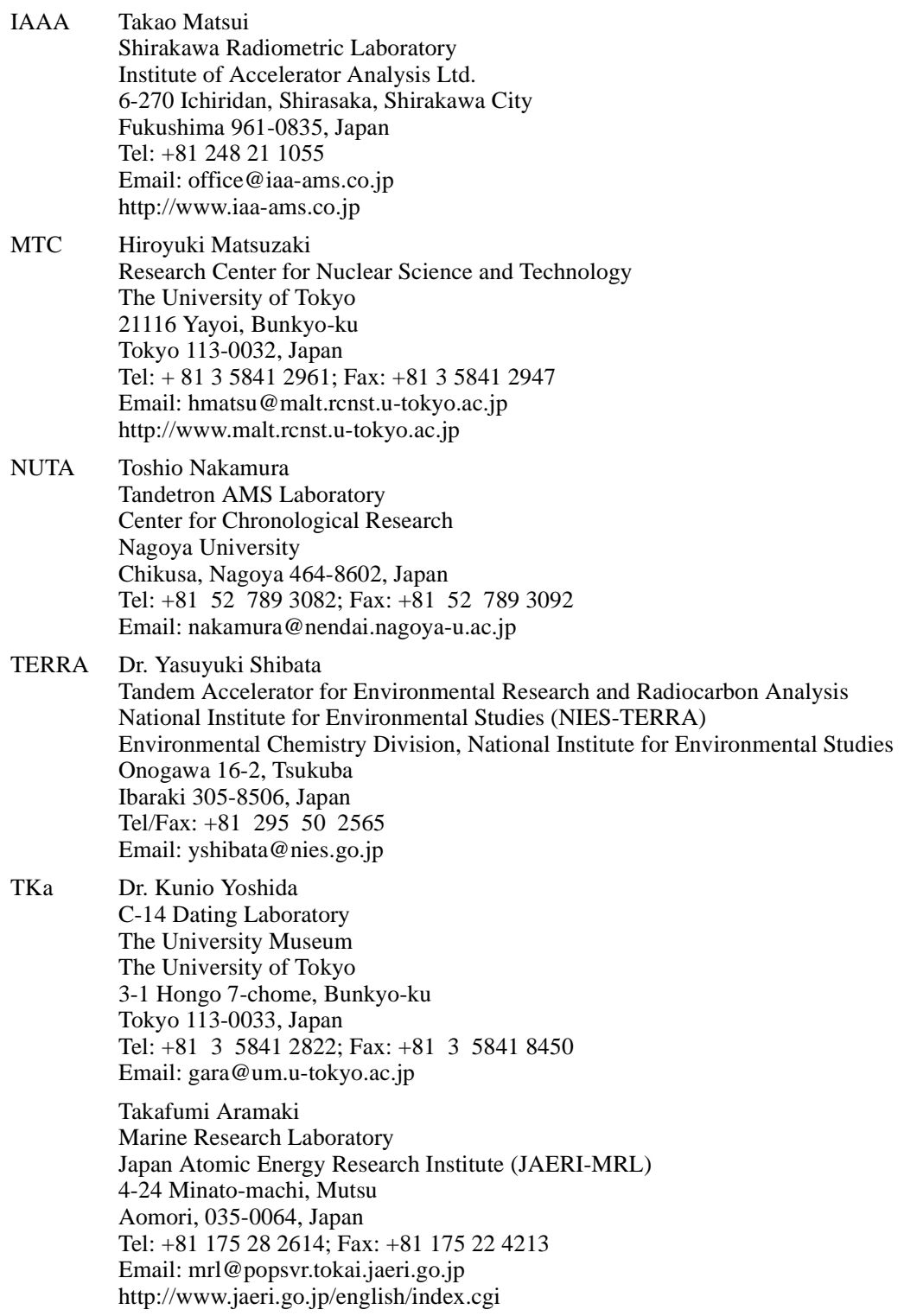

\section{KOREA}

SNU Jong-Chan Kim

The Inter-University Center for National Science Research Facility

Seoul National University

Seoul 151-742, Korea

Tel: +82 2880 6593; Fax: +82 28843092

Email: jckim@phya.snu.ac.kr

http://phya.snu.ac.kr/english/ 


\section{THE NETHERLANDS}

GrA J. van der Plicht

Centre for Isotope Research

University of Groningen

Nijenborgh 4

NL-9747 AG Groningen, the Netherlands

Tel: +31 50 3634760; Fax: +31 503634738

Email: j.van.der.plicht@ rug.nl

UtC K. van der Borg

R. J. van de Graaff Laboratorium

Universiteit Utrecht

Princetonplein 5

P.O. Box 80000

NL-3504 CC Utrecht, the Netherlands

Tel: +31 30532238 or +313053 1492; Fax: +31 302532532

Email: k.vanderborg@phys.uu.nl

\section{NEW ZEALAND}

NZA Christine Prior

Rafter Radiocarbon Laboratory

Institute of Geological and Nuclear Sciences, Ltd.

P.O. Box 31-312

Lower Hutt, New Zealand

Tel: +64 4570 4644; Fax: +64 45704657

Email: C.Prior@gns.cri.nz

http://www.RafterRadiocarbon.co.nz

\section{POLAND}

Poz

Dr. Tomasz Goslar

Poznań Radiocarbon Laboratory

Foundation of the Adam Mickiewicz University

ul. Rubież 46

61-612 Poznań, Poland

Tel: + 0618279782

Email: c14@ radiocarbon.pl

http://www.radiocarbon.pl

SPAIN

CNA Francisco Javier Santos Arévalo

Centro Nacional de Aceleradores (CNA)

Avda. Thomas Alba Edison, $\mathrm{n}^{\circ} 7$

Isla de la Cartuja

41092 Sevilla, Spain

Tel: +34 9544605 53; Fax: +34 954460145

Email: fsantos@us.es

http://www.centro.us.es/cna

\section{SWEDEN}

LuA

Göran Skog

Radiocarbon Dating Laboratory

University of Lund

Tornavägen 13

SE-223 63 Lund, Sweden

Tel: +46 46222 7885; Fax: +46 462224830

Email: Goran.Skog@C14lab.lu.se

Ua Göran Possnert

Tandem Laboratory

University of Uppsala, Box 529

SE-751 20 Uppsala, Sweden

Tel: +46 18 4713059; Fax: +46 18555736

Email: Goran.Possnert@ Angstrom.uu.se 


\section{SWITZERLAND}

ETH Georges Bonani

ETH/AMS Facility

Institut für Teilchenphysik

Eidgenössische Technische Hochschule Hönggerberg

CH-8093 Zürich, Switzerland

Tel: +41 1633 2041; Fax: +41 16331067

Email: bonani@phys.ethz.ch

\section{UNITED KINGDOM}

OxA C. Bronk Ramsey and T. F. G. Higham

Oxford Radiocarbon Accelerator Unit

Research Laboratory for Archaeology and the History of Art

Oxford University

Dyson Perrins Building, South Parks Road

Oxford OX1 3QY, United Kingdom

Tel: +44 1865 285229; Fax: +44 1865285220

Email: orau@ rlaha.ox.ac.uk

http://c14.arch.ox.ac.uk

SUERC Tony Fallick (Director of SUERC), Gordon Cook (Head of SUERC ${ }^{14} \mathrm{C}$ Laboratory),

Charlotte Bryant (Head of NERC ${ }^{14} \mathrm{C}$ Laboratory), Stewart Freeman (Head of SUERC AMS Team)

Scottish Universities Environmental Research Centre

Scottish Enterprise Technology Park

Rankine Avenue

East Kilbride G75 0QF, Scotland

Tel: +44 1355 223332; Fax: +44 1355229898

http://www.gla.ac.uk/suerc

UBA Dr. Paula Reimer and Prof. Gerry McCormac

14CHRONO Centre for Climate, the Environment, and Chronology

School of Geography, Archaeology, and Palaeoecology

The Queen's University of Belfast

42 Fitzwilliam Street

Belfast BT9 6AX, United Kingdom

Tel: +44 2890 973980; Fax: +44 2890973897

Email: p.j.reimer@qub.ac.uk

http://www.chrono.qub.ac.uk; http://www.qub.ac.uk/arcpal

\section{UNITED STATES}

AA

A. J. Timothy Jull

NSF-Arizona AMS Facility

1118 E. Fourth Street

P.O. Box 210081

The University of Arizona

Tucson, Arizona 85721-0081, USA

Tel: +1 520621 6810; Fax: +1 5206219619

Email: ams@ physics.arizona.edu

http://www.physics.arizona.edu/ams

Beta D. G. Hood

Beta Analytic, Inc.

Professional Radiocarbon Dating Services

4985 SW 74 Court

Miami, Florida 33155, USA

Tel: +1 305667 5167; Fax: +1 3056630964

Email: beta@ radiocarbon.com

http://www.radiocarbon.com/

CAMS John Knezovich

Center for Accelerator Mass Spectrometry

Lawrence Livermore National Laboratory

P.O. Box 808, L-397

Livermore, California 94550, USA

Tel: +1 510422 9670; Fax: +1 5104237884

Email: knezovich1@1lnl.gov 
KCCAMS John Southon, Ellen Druffel, Susan Trumbore, Guaciara M. dos Santos Earth System Science Department

B321 Croul Hall, University of California, Irvine

Irvine, California 92697-3100, USA

Tel: +1 (949) 824 3674; Fax: +1 (949) 8243874

Email: jsouthon@uci.edu and/or gdossant@uci.edu http://www.ess.uci.edu/ams/

NSRL Scott Lehman

INSTAAR Laboratory for AMS Radiocarbon Preparation and Research (NSRL)

CU-Boulder

1560 30th St.

Campus Box 450

Boulder, Colorado 80309-0450, USA

Tel: +1 303492 0362; Fax: +1 3034926388

Email: Scott.Lehman@colorado.edu

http://www.colorado.edu/INSTAAR/RadiocarbonDatingLab/

PL Prof. Marc Caffee

Purdue Rare Isotope Measurement Laboratory

Purdue University

525 Northwestern Avenue

West Lafayette, Indiana 47907-2036, USA

Tel: +1 765494 5381; Fax: +1 7654967228

Email: mcaffee@physics.purdue.edu

http://primelab.physics.purdue.edu

PRI Linda Scott Cummings

PaleoResearch Institute

2675 Youngfield St

Golden, Colorado 80401, USA

Tel: +1 303277 9848; Fax: +1 3034622700

Email: ams@paleoresearch.com

http://www.paleoresearch.com

UGa Dr. John E. Noakes

Center for Applied Isotope Studies

The University of Georgia

120 Riverbend Road

Athens, Georgia 30602-4702, USA

Tel: +1 706542 1395; Fax: +1 7065426106

Email: jenoakes@uga.edu

http://www.cais.uga.edu

WHAMS John M. Hayes

National Ocean Sciences AMS Facility

Woods Hole Oceanographic Institution

McLean Laboratory, Mail Stop \#8

Woods Hole, Massachusetts 02543-1539, USA

Tel: +1 508289 3345; Fax: +1 5084572183

Email: jhayes@whoi.edu

http://nosams.whoi.edu 


\begin{tabular}{|c|c|c|c|c|c|}
\hline \multicolumn{3}{|c|}{ LABORATORIES - CODE DESIGNATIONS* } & \multirow{2}{*}{$\begin{array}{l}\text { DK } \\
\text { DRI* }\end{array}$} & \multirow{2}{*}{$\begin{array}{l}\text { Univ. de Dakar } \\
\text { Desert Research Institute }\end{array}$} & \multirow{2}{*}{$\begin{array}{l}\text { Sénégal } \\
\text { USA }\end{array}$} \\
\hline A & University of Arizona & USA & & & \\
\hline AA & NSF-Ariz. AMS Facility & USA & DSA & CIRCE, Caserta & Italy \\
\hline AAR & University of Aarhus & Denmark & ENEA & ENEA, Bologna & Italy \\
\hline $\mathrm{AC}$ & Ingeis & Argentina & Erl & Erlangen AMS Facility & Germany \\
\hline \multirow[t]{2}{*}{$\mathrm{AECV}^{*}$} & \multirow{2}{*}{$\begin{array}{l}\text { Alberta Environmental } \\
\text { Center of Vegreville }\end{array}$} & \multirow[t]{2}{*}{ Canada } & ETH & ETH/AMS Facility & Switzerland \\
\hline & & & $\mathrm{F}^{*}$ & Florence & Italy \\
\hline AERIK* & Atomic Energy Res. Inst. & Korea & $\mathrm{Fr}^{*}$ & Freiberg & Germany \\
\hline $\mathrm{ALG}^{*}$ & Algiers & Algeria & Fra* & Frankfurt & Germany \\
\hline ANL* & Argonne Nat. Lab. & USA & FSU* & Florida State University & USA \\
\hline \multirow[t]{2}{*}{ ANSTO } & \multirow{2}{*}{$\begin{array}{l}\text { Australian Nuclear } \\
\text { Science \& Technology }\end{array}$} & \multirow[t]{2}{*}{ Australia } & $\mathrm{FZ}$ & Fortaleza & Brazil \\
\hline & & & $\mathrm{G}^{*}$ & Göteborg & Sweden \\
\hline ANTW* & \multirow{2}{*}{$\begin{array}{l}\text { Antwerp } \\
\text { Australian National } \\
\text { University }\end{array}$} & \multirow{2}{*}{$\begin{array}{l}\text { Belgium } \\
\text { Australia }\end{array}$} & $\mathrm{GAK}^{*}$ & Gakushuin University & Japan \\
\hline ANU* & & & Gd & Gliwice & Poland \\
\hline ANUA & ANU Accelerator & Australia & GD* & Gdansk & Poland \\
\hline \multirow{2}{*}{$\begin{array}{l}\mathrm{AU}^{*} \\
\mathrm{~B}\end{array}$} & University of Alaska & USA & Gif & Gif-sur-Yvette & France \\
\hline & & Switzerland & Gif A & Gif-sur-Yvette and Orsay & France \\
\hline $\mathrm{Ba}$ & Bratislava & Slovakia & GIN & Geological Institute & Russia \\
\hline $\mathrm{BC}^{*}$ & Brooklyn College & USA & GL* & Geochronological Lab. & England \\
\hline Beta & Beta Analytic & USA & Gro* & Groningen & the Netherlands \\
\hline BGS & Brock University & Canada & $\mathrm{GrN}$ & Groningen & the Netherlands \\
\hline BIOCAMS* & Miami & USA & GrA & Groningen AMS & the Netherlands \\
\hline Birm* & Birmingham & UK & GSC & Geological Survey & Canada \\
\hline Bln & Berlin & Germany & GU & Scottish Universities & Scotland \\
\hline $\mathrm{BM} *$ & British Museum & England & & Research \& Reactor Centr & \\
\hline BONN* & Universität Bonn & Germany & GX & Geochron Laboratories & USA \\
\hline BS & Birbal Sahni Institute & India & $\mathrm{H}^{*}$ & Heidelberg & Germany \\
\hline $\mathrm{C}^{*}$ & Chicago & USA & HAM & Hamburg & Germany \\
\hline CAMS & Center for Accelerator & USA & $\mathrm{HAR}^{*}$ & Harwell & England \\
\hline & Mass Spectrometry & & Hd & Heidelberg & Germany \\
\hline CAR* & Univ. College, Cardiff & Wales & Hel & Helsinki & Finland \\
\hline CENA & Centro Energia & Brazil & $\mathrm{HIG}^{*}$ & Hawaii Inst. of Geophys. & USA \\
\hline & Nuclear na Agricultura & Drazil & $\mathrm{HL}$ & $\begin{array}{l}\text { Second Institute of } \\
\text { Oceanography }\end{array}$ & China \\
\hline CG & Institute of Geology & China & HNS* & Hasleton-Nuclear, & USA \\
\hline $\mathrm{CH}^{*}$ & Chemistry Laboratory & India & & Palo Alto, California & \\
\hline CNA & Centro Nacional de & Spain & $\mathrm{Hv}$ & Hannover & Germany \\
\hline & Aceleradores & & $I^{*}$ & Teledyne Isotopes & USA \\
\hline CRCA* & Cairo & Egypt & IAA & Institute of Accelerator & Japan \\
\hline CRL & $\begin{array}{l}\text { Czech Radiocarbon } \\
\text { Laboratory }\end{array}$ & Czech Republic & & Analysis (beta counting) & \\
\hline CSIC & $\begin{array}{l}\text { Geochronology Lab, } \\
\text { IQFR-CSIC, Madrid }\end{array}$ & Spain & IAAA & $\begin{array}{l}\text { Institute of Accelerator } \\
\text { Analysis (AMS) }\end{array}$ & Japan \\
\hline $\mathrm{CSM}^{*}$ & $\begin{array}{l}\text { Cosmochemistry Lab. } \\
\text { USSR Academy of Scienc }\end{array}$ & $\begin{array}{l}\text { USSR } \\
\text { ces }\end{array}$ & IAEA & $\begin{array}{l}\text { International Atomic } \\
\text { Energy Agency }\end{array}$ & Austria \\
\hline $\mathrm{CT}^{*}$ & Caltech, Calif. Inst. Tech. & USA & $\begin{array}{l}\text { IAEA- } \\
\text { MEL }\end{array}$ & Marine Environmental & Monaco \\
\hline $\mathrm{CU}$ & Charles University & Czech Republic & & Laboratory & \\
\hline $\mathrm{D}^{*}$ & Dublin, Trinity College & Ireland & ICEN* & $\begin{array}{l}\text { Instituto Tecnológico } \\
\text { e Nuclear }\end{array}$ & Portugal \\
\hline $\begin{array}{l}\text { Dak* }^{*} \\
\text { DAL* }^{*}\end{array}$ & $\begin{array}{l}\text { Univ. of Dakar } \\
\text { Dalhousie University }\end{array}$ & $\begin{array}{l}\text { Sénégal } \\
\text { Canada }\end{array}$ & IEMAE & $\begin{array}{l}\text { Institute of Evolutionary } \\
\text { Morphology and Animal }\end{array}$ & Russia \\
\hline $\mathrm{DE}^{*}$ & USGS, Denver & USA & & Ecology & \\
\hline Deb & Debrecen & Hungary & IFAO & Institut français & Egypt \\
\hline DEM & NCSR Demokritos & Greece & & d'archéologie orientale & \\
\hline DIC* & Dicar Corp and Dicarb & USA & IGAN & Institute of Geography & Russia \\
\hline & Radioisotope Company & & IGS* & Inst. of Geological Sci. & Sweden \\
\hline
\end{tabular}

* Indicates laboratories that are closed, no longer measuring ${ }^{14} \mathrm{C}$, or operating under a different code designation. 


\begin{tabular}{|c|c|c|c|c|c|}
\hline IGSB & Inst. of Geological Sci. & Belarus & $\mathrm{Ma}^{*}$ & University of Winnepeg & Canada \\
\hline IHME & $\begin{array}{l}\text { Marzeev Inst. of Hygiene } \\
\text { and Medical Ecology }\end{array}$ & Ukraine & MAG & $\begin{array}{l}\text { Quaternary Geology and } \\
\text { Geochronology Laborator }\end{array}$ & $\begin{array}{l}\text { Russia } \\
\text { y }\end{array}$ \\
\hline \multirow{3}{*}{$\begin{array}{l}\text { II }^{*} \\
\text { IMTA }\end{array}$} & Isotopes, Inc., Palo Alto & USA & $\mathrm{MC}^{*}$ & Centre Scientifique de & Monaco \\
\hline & Inst. Mexicano de & & & Monaco & \\
\hline & Tecnología del Agua & Mexico & METU & Middle East Technical & Turkey \\
\hline \multirow[t]{2}{*}{ IOAN* } & Institute of Oceano- & Russia & & University & \\
\hline & graphy & & ML* & Miami & USA \\
\hline \multirow{3}{*}{$\begin{array}{l}\text { IORAN* } \\
\text { IRPA }\end{array}$} & Institute of Oceanology & Russia & Mo* & Verdanski Inst. of & USSR \\
\hline & Royal Institute for & Belgium & & Geochemistry, Moscow & \\
\hline & Cultural Heritage & ISA & MOC* & $\begin{array}{l}\text { Archaeological Institute, } \\
\text { Czechoslovak Acad. of Sc }\end{array}$ & $\begin{array}{l}\text { Czech Republic } \\
\text { i. }\end{array}$ \\
\hline ISGS & Geological Survey & ה & $\mathrm{MP}^{*}$ & Magnolia Petroleum & USA \\
\hline \multirow{4}{*}{$\begin{array}{l}\text { IVAN* } \\
\text { IVIC* } \\
\text { IWP* }\end{array}$} & Institute of Volcanology & Ukraine & MRRI* & Marine Resources & USA \\
\hline & Caracas & Venezuela & & Research Institute & \\
\hline & Institute of Water & Russia & MSU* & Moscow State University & Russia \\
\hline & Problems & & MTC & University of Tokyo & Japan \\
\hline \multirow[t]{2}{*}{$\mathrm{J}$} & Max-Planck-Institut & Germany & $\mathrm{N}^{*}$ & Nishina Memorial & Japan \\
\hline & $\begin{array}{l}\text { für Biogeochemie, Jena } \\
\text { Geological Survey of }\end{array}$ & Japan & NIST & $\begin{array}{l}\text { National Institute of } \\
\text { Standards and Technology }\end{array}$ & USA \\
\hline JGS* & Japan & & NPL* & National Physical & England \\
\hline \multirow[t]{2}{*}{ JUBR } & Biren Roy Research & India & & Laboratory, Middlesex & \\
\hline & Laboratory & & NS* & Nova Scotia Research & Canada \\
\hline $\mathrm{K}$ & National Museum & Denmark & & Foundation & \\
\hline KAERI* & $\begin{array}{l}\text { Korean Atomic Energy } \\
\text { Research Institute }\end{array}$ & Korea & NSRL & $\begin{array}{l}\text { INSTAAR - University } \\
\text { of Colorado }\end{array}$ & USA \\
\hline $\mathrm{KCP}$ & $\begin{array}{l}\text { National Cultural } \\
\text { Property Research } \\
\text { Institute }\end{array}$ & Korea & NSTF* & $\begin{array}{l}\text { Nuclear Science and } \\
\text { Technology Facility, } \\
\text { State Univ. of New York }\end{array}$ & USA \\
\hline \multirow[t]{2}{*}{ KEEA } & Kyushu Environmental & Japan & $\mathrm{NSW}^{*}$ & U. of New South Wales & Australia \\
\hline & Evaluation Association & & NTU & National Taiwan & Republic of China \\
\hline KI & Kiel & Germany & & University & \\
\hline KIA & Kiel AMS & Germany & NU & Nihon University & Japan \\
\hline \multirow{2}{*}{ Ki (KIEV) } & Institute of Radio- & Ukraine & NUTA & Tandetron AMS Lab & Japan \\
\hline & $\begin{array}{l}\text { Geochemistry of the } \\
\text { Environment }\end{array}$ & & $\mathrm{Ny} *$ & $\begin{array}{l}\text { Nancy, Centre de } \\
\text { Recherches Radiogéologic }\end{array}$ & $\begin{array}{l}\text { France } \\
\text { ques }\end{array}$ \\
\hline \multirow[t]{2}{*}{ KIK } & Royal Institute for & Belgium & NZ* & Rafter Radiocarbon Lab & New Zealand \\
\hline & Cultural Heritage & & NZA & Rafter (AMS) & New Zealand \\
\hline $\mathrm{KN}$ & Köln & Germany & $\mathrm{O}^{*}$ & Humble Oil \& Refining & USA \\
\hline KR & Krakow & Poland & OBDY* & ORSTOM Bondy & France \\
\hline \multirow{2}{*}{$\begin{array}{l}\mathrm{KRIL}^{*} \\
\mathrm{KSU}^{*}\end{array}$} & Krasnoyarsk Institute & Russia & OR & Research Center of & Japan \\
\hline & Kyoto Sangyo University & Japan & & Radioisotopes & \\
\hline \multirow{2}{*}{$\begin{array}{l}\mathrm{L} * \\
\mathrm{LAR}^{*}\end{array}$} & Lamont-Doherty & USA & ORINS* & Oak Ridge Institute & USA \\
\hline & Liège State University & Belgium & & of Nuclear Studies & \\
\hline LE & St. Petersburg & Russia & OWU* & Ohio Wesleyan Univ. & USA \\
\hline \multirow{2}{*}{$\begin{array}{l}\mathrm{LIH} \\
\mathrm{LJ}^{*}\end{array}$} & NCSR Demokritos & Greece & $\mathrm{OX}^{*}$ & USDA & USA \\
\hline & Scripps (UCSD) La Jolla & USA & & Oxford, Mississippi & \\
\hline LOD & Łódź & Poland & OxA & Oxford Radiocarbon & England \\
\hline LP & La Plata & Argentina & $\mathrm{OZ}$ & $\begin{array}{l}\text { Accelerator Unit } \\
\text { ANSTO-ANTARES }\end{array}$ & Australia \\
\hline \multirow{2}{*}{$\begin{array}{l}\text { LTL } \\
\text { Lu* }^{*}\end{array}$} & University of Lecce & Italy & $\mathrm{P}^{*}$ & Univ. of Pennsylvania & USA \\
\hline & Lund & Sweden & PAL & Palynosurvery Co. & Japan \\
\hline LuS & Lund & Sweden & $\mathrm{Pi}^{*}$ & Pisa & Italy \\
\hline LU & St. Petersburg State & Russia & $\mathrm{PI}^{*}$ & Permafrost Institute & Russia \\
\hline & University & & PIC* & Packard & USA \\
\hline $\mathrm{Lv}^{*}$ & Louvain-la-Neuve & Belgium & PITT* & University of Pittsburgh & USA \\
\hline Ly & University of Lyon & France & Poz & Poznań & Poland \\
\hline $\mathrm{LZ}$ & Umweltforschungs- & Germany & Pr* & Prague & Czech Republic \\
\hline$M^{*}$ & University of Michigan & USA & PKU & Peking University & China \\
\hline
\end{tabular}




\begin{tabular}{|c|c|c|}
\hline PKUAMS & Peking Univ. AMS lab & China \\
\hline PL & $\begin{array}{l}\text { Purdue Rare Isotope } \\
\text { Measurement Laboratory }\end{array}$ & USA \\
\hline PLD & Paleo Labo. Co., Ltd. & Japan \\
\hline PRI & PaleoResearch Inst. & USA \\
\hline PRL & $\begin{array}{l}\text { Radiocarbon Dating } \\
\text { Research Unit }\end{array}$ & India \\
\hline PRLCH & $\begin{array}{l}\text { Physical Research } \\
\text { Laboratory }\end{array}$ & India \\
\hline PSU* & Pennsylvania State Univ. & USA \\
\hline Pta & Pretoria & South Africa \\
\hline Q & Cambridge & England \\
\hline QL* & $\begin{array}{l}\text { Quaternary Isotope } \\
\text { Laboratory }\end{array}$ & USA \\
\hline QC* & Queens College & USA \\
\hline QU* & $\begin{array}{l}\text { Centre de Recherches } \\
\text { Minérales, Québec }\end{array}$ & Canada \\
\hline $\mathrm{R}$ & Rome & Italy \\
\hline $\mathrm{RCD}$ & Radiocarbon Dating & England \\
\hline RI* & Radiochemistry, Inc. & USA \\
\hline RIDDL* & Simon Fraser Univ. & Canada \\
\hline Riga & Institute of Science & Latvia \\
\hline RL* & Radiocarbon, Ltd. & USA \\
\hline Rome & $\begin{array}{l}\text { Dept. of Earth } \\
\text { Sciences, Rome }\end{array}$ & Italy \\
\hline RT & Rehovot & Israel \\
\hline $\mathrm{RU}^{*}$ & Rice University & USA \\
\hline S* & Saskatchewan & Canada \\
\hline $\mathrm{Sa}^{*}$ & Saclay, Gif-sur-Yvette & France \\
\hline Sac & $\begin{array}{l}\text { Instituto Tecnológico } \\
\text { e Nuclear }\end{array}$ & Portugal \\
\hline $\mathrm{SacA}$ & Gif sur Yvette (Saclay) & France \\
\hline SFU* & Simon Fraser Univ. & Canada \\
\hline $\mathrm{Sh}^{*}$ & Shell Development Co. & USA \\
\hline $\mathrm{SI}^{*}$ & Smithsonian Institution & USA \\
\hline $\mathrm{SL}^{*}$ & Sharp Laboratories & USA \\
\hline $\mathrm{SM}^{*}$ & Mobil Oil Corp., Dallas & USA \\
\hline SMU* & Southern Methodist Univ. & USA \\
\hline SNU & Seoul National Univ. & Korea \\
\hline SOAN & $\begin{array}{l}\text { Institute of Geology, } \\
\text { Geophysics, and } \\
\text { Mineralogy }\end{array}$ & Russia \\
\hline $\mathrm{SR}^{*}$ & Salisbury, Rhodesia & Rhodesia \\
\hline SRR & $\begin{array}{l}\text { NERC Radiocarbon } \\
\text { Laboratory }\end{array}$ & Scotland \\
\hline $\mathrm{St}^{*}$ & Stockholm & Sweden \\
\hline $\mathrm{Su}^{*}$ & Finland & Finland \\
\hline SUA* & University of Sydney & Australia \\
\hline SUERC & $\begin{array}{l}\text { Scottish Universities } \\
\text { Environmental } \\
\text { Research Centre }\end{array}$ & Scotland \\
\hline SWA & Swansea & Wales \\
\hline $\mathrm{T}$ & Trondheim & Norway \\
\hline $\mathrm{Ta}$ & Tartu & Estonia \\
\hline TAM* & Texas A \& M University & USA \\
\hline $\mathrm{TB}$ & Tblisi & Georgia \\
\hline TBNC* & Kaman Instruments & USA \\
\hline TEM* & Temple University & USA \\
\hline
\end{tabular}

\begin{tabular}{|c|c|c|}
\hline $\mathrm{TF}^{*}$ & $\begin{array}{l}\text { Tata Institute of } \\
\text { Fundamental Research }\end{array}$ & India \\
\hline TK & University of Tokyo & Japan \\
\hline TKa & Univ. of Tokyo-AMS & Japan \\
\hline TKU & Turku & Finland \\
\hline $\operatorname{Tln}$ & Tallinn & Estonia \\
\hline TO & IsoTrace Laboratory & Canada \\
\hline TUNC* & $\begin{array}{l}\text { Tehran University } \\
\text { Nuclear Centre }\end{array}$ & Iran \\
\hline Tx* & Texas & USA \\
\hline $\mathrm{U}$ & Uppsala University & Sweden \\
\hline $\mathrm{Ua}$ & Uppsala AMS & Sweden \\
\hline $\mathrm{UB}^{*}$ & Belfast & Northern Ireland \\
\hline UBA & Belfast & Northern Ireland \\
\hline UBAR & University of Barcelona & Spain \\
\hline UCD & $\begin{array}{l}\text { University College, } \\
\text { Dublin }\end{array}$ & Ireland \\
\hline UCI & $\begin{array}{l}\text { University of California, } \\
\text { Irvine }\end{array}$ & USA \\
\hline UCLA* & $\begin{array}{l}\text { University of California, } \\
\text { Los Angeles }\end{array}$ & USA \\
\hline UCR* & $\begin{array}{l}\text { University of California, } \\
\text { Riverside }\end{array}$ & USA \\
\hline UD* & Udine & Italy \\
\hline UGa & University of Georgia & USA \\
\hline UGRA & University of Granada & Spain \\
\hline UL & University of Laval & Canada \\
\hline $\mathrm{UM}^{*}$ & University of Miami & USA \\
\hline UNAM & $\begin{array}{l}\text { National Autonomous } \\
\text { University of Mexico }\end{array}$ & Mexico \\
\hline $\mathrm{UQ}^{*}$ & $\begin{array}{l}\text { University of Quebec } \\
\text { at Montréal }\end{array}$ & Canada \\
\hline URCRM* & $\begin{array}{l}\text { Ukrainian Research Ctr. } \\
\text { for Radiation Medicine }\end{array}$ & Ukraine \\
\hline URU & University of Uruguay & Uruguay \\
\hline USGS* & USGS, Menlo Park & USA \\
\hline UtC & $\begin{array}{l}\text { Utrecht van de Graaff } \\
\text { Laboratorium }\end{array}$ & the Netherlands \\
\hline UTCAG & University of Tennessee & USA \\
\hline $\mathrm{UW}^{*}$ & University of Washington & USA \\
\hline $\mathrm{V}^{*}$ & Melbourne, Victoria & Australia \\
\hline VERA & $\begin{array}{l}\text { Institut für Radiumfor- } \\
\text { schung und Kernphysik }\end{array}$ & Austria \\
\hline VRI* & Universität Wien & Austria \\
\hline $\mathrm{W}^{*}$ & USGS, National Center & USA \\
\hline WAT $^{*}$ & University of Waterloo & Canada \\
\hline WHAMS & $\begin{array}{l}\text { National Ocean Sciences } \\
\text { AMS Facility }\end{array}$ & USA \\
\hline WIS* & Univ. of Wisconsin & USA \\
\hline Wk & University of Waikato & New Zealand \\
\hline WRD* & USGS Washington, D.C. & USA \\
\hline WSU* & Washington State Univ. & USA \\
\hline XLLQ & $\begin{array}{l}\text { Xi'an Lab. ofChina } \\
\text { Loess and Quaternary } \\
\text { Geology }\end{array}$ & China \\
\hline $\mathrm{X}^{*}$ & Whitworth College & USA \\
\hline $\mathrm{Y}^{*}$ & Yale University & USA \\
\hline $\mathrm{Ya}^{*}$ & Yale University & USA \\
\hline $\mathrm{Z}$ & Zagreb & Croatia \\
\hline
\end{tabular}

